\title{
Parcours universitaires des sages-femmes enseignantes et directrices des écoles de sages-femmes en France : enquête descriptive*
}

\author{
Academic background of midwifery teachers and education heads in France \\ - a descriptive survey
}

\author{
Christine MORIN ${ }^{1}$ et Marie-Christine LEYMARIE ${ }^{2}$ \\ 1 Sage-femme enseignante Ecole de sages-femmes, Université et centre hospitalier universitaire de Bordeaux (France). \\ Présidente de la Conférence nationale des enseignants en maïeutique (2013-2017) \\ 2 Université Clermont-Auvergne et Ecole de sages-femmes, Centre hospitalier universitaire de Clermont-Ferrand, France
}

Manuscrit soumis le 30 novembre 2015 ; commentaires éditoriaux formulés aux auteurs le 8 septembre 2016 ; accepté pour publication le $1^{\mathrm{er}}$ février 2017

Mots-clés
Ecoles de sages-
femmes ; France ;
sage-femme
enseignante ;
qualification ;
université ; recherche
maïeutique;processus
de Bologne ; licence-
master-doctorat

Résumé - Contexte : Les exigences académiques pour les sages-femmes enseignantes ne sont pas les mêmes en France que celles d'autres pays européens, comme le Royaume Uni et la Suède, par exemple. Malgré l'harmonisation européenne des formations de l'enseignement supérieur qui s'est traduite en France par une organisation des études de sages-femmes en cinq ans, la formation des sages-femmes enseignantes n'a pas encore été accompagnée d'exigences universitaires. Il n'existe aucune donnée nationale sur le sujet. Objectif : Décrire les diplômes universitaires détenus par les sages-femmes enseignantes et directrices des écoles de sagesfemmes en France en 2014. Méthode : Enquête par questionnaire envoyé par messagerie électronique aux directrices des écoles de sages-femmes afin d'être rempli par elles-mêmes et les enseignantes. Participants : Sages-femmes enseignantes et directrices. Résultats : Les 241 (100\%) sages-femmes enseignantes et directrices en activité dans les écoles de sages-femmes ont participé à l'enquête. La moitié des sages-femmes directrices (17/34) et 88 sur 207 (42,5\%) sages-femmes enseignantes possédaient un diplôme de master. Une enseignante et une directrice étaient titulaires d'un doctorat. Au moment de l'étude, $37(15,3 \%)$ sages-femmes enseignantes ou directrices suivaient un programme de master et $20(8,3 \%)$ un programme de doctorat dans des disciplines variées (sociologie, santé publique/épidémiologie, philosophie, sciences de l'éducation, droit, management/gestion). Un total de $142(58,9 \%)$ sages-femmes enseignantes ou directrices avaient validé ou suivaient un programme de master et $22(9,1 \%)$ étaient titulaires ou suivaient un programme de doctorat. Discussion : Il s'agit de la première

\footnotetext{
* Note éditoriale : Dans le but d'alléger le texte, le genre féminin a été employé bien que les propos s'appliquent autant aux hommes sages-femmes exerçant les fonctions de direction ou d'enseignement; moins de $5 \%$ de sages-femmes enseignantes sont des hommes et aucun n'exerce des fonctions de direction.
} 


\section{Keywords}

French midwifery schools; midwifery teachers, academic qualifications, university, midwifery research, maieutic research; undergraduatemaster's-PhD degree enquête nationale décrivant le niveau académique des sages-femmes enseignantes françaises. Elle s'inscrit dans le cadre des besoins des sages-femmes françaises qui souhaitent une meilleure reconnaissance de leur profession et de leur rôle. Cet état des lieux, du niveau académique du corps enseignant sage-femme exerçant dans les écoles, apporte des éléments utiles pour la demande d'intégration des écoles à l'université sous forme de composantes autonomes ainsi que pour la reconnaissance d'un statut d'enseignant universitaire en maïeutique. Conclusion : Cette enquête a permis de décrire le parcours académique des sages-femmes enseignantes en France. Le nombre de sages-femmes enseignantes titulaires d'un doctorat est faible comparé à celui du Royaume Uni ou de la Suède.

Abstract - Background: Academic requirements for French midwifery teachers are somewhat different in France than in other European countries, e.g. United Kingdom and Sweden. Despite the harmonization of higher education programs that led to a five-year education program for midwives in France, midwifery teacher training has not been associated with specific academic requirements and no data are as yet available regarding such requirements. Objective: To describe the degrees held by midwifery teachers and education heads in France in 2014. Method: E-mail questionnaires sent to all heads of midwifery education for completion by midwifery heads and teachers. Participants: All midwifery teachers and education heads in France. Results: 241 (100\%) midwifery teachers and education heads employed in the schools took part in the study. $50 \%$ of midwifery education heads $(17 / 34)$ and $42.5 \%$ (88/207) of midwifery teachers held a master's degree. One teacher and one head had a PhD. At the time of the study, $37(15.3 \%)$ of all participants were studying for a master's degree and $20(8.3 \%)$ for a $\mathrm{PhD}$ in various fields (sociology, public health/epidemiology, philosophy, educational sciences, law, management). 142 (58.9\%) participants were studying for or held a master's and $22(9.1 \%)$ were studying for or held a PhD. Discussion: This is the first national study describing the academic degrees of French midwifery teachers. The study is part of the current appeals by French midwives for better recognition of their profession and role. The assessment of the academic degrees of midwifery teachers currently practicing in midwifery schools provides some useful information to support the calls for integrating midwifery education as stand-alone units as well as recognizing a university lecturer status. Conclusion: The survey helped describe the academic background of midwifery teachers in France. The number of midwifery teachers holding a $\mathrm{PhD}$ remains low compared to the United Kingdom or Sweden.

\section{Introduction}

\section{Contexte}

Depuis plus de trente ans, de nombreux travaux de recherche clinique, dédiés à la santé maternelle et néonatale, ont été menés par des équipes de chercheurs pluridisciplinaires comprenant des sages-femmes $^{[1-5]}$. En Europe, en 2010, le projet EURO-PERISTAT, qui avait pour objectif de suivre et évaluer la santé des mères et des enfants durant la période périnatale en utilisant des indicateurs valides et fiables, a situé la France dans une position moyenne ${ }^{[6]}$. Ce rapport attribuait de meilleurs résultats à la Suède et au Royaume-Uni concernant la prématurité ou le ratio de mortalité maternelle durant la période 2006-2010, par exemple. Concernant la mortalité néonatale (décès dans les 27 premiers jours après la naissance), le Royaume Uni et la France présentaient sensiblement les mêmes résultats mais ceux-ci restaient inférieurs à ceux de la Suède.

Une des caractéristiques de l'évolution de la profession de sage-femme réside dans la qualité et l'efficacité des soins apportés aux mères et aux familles dans le monde entier ${ }^{[7]}$. Spécialistes de la grossesse 
normale (ou physiologique), les sages-femmes ont un rôle inestimable pour améliorer la santé des femmes et des nouveau-nés, quel que soit le niveau économique d'un pays, comme cela a été mis en évidence dans la revue scientifique The Lancet ${ }^{[5,8,9]}$.

En France, en position centrale pour la « surveillance et l'encadrement de la fonction maternelle », les sages-femmes ont contribué au XIXe siècle à la diffusion de l'hygiène pasteurienne ${ }^{[10]}$ et à la diminution de la mortalité maternelle et infantile ${ }^{[11]}$. Le développement de l'activité salariée au détriment de l'exercice libéral, en insérant les sages-femmes au sein de la hiérarchie médicale hospitalière, a profondément modifié leur rôle et leur fonction ${ }^{[12]}$. La surveillance de la femme enceinte à faible risque tout au long du travail et l'accouchement eutocique (normal) sont restés dans le champ de la compétence des sagesfemmes. Les gynécologues obstétriciens, chargés des accouchements dystociques, ont obtenu la responsabilité des services de maternité ${ }^{[12]}$. Cependant, les compétences des sages-femmes ont été élargies avec, par exemple, l'habilitation à la pratique officielle des échographies depuis janvier $1986^{[12]}$ et leurs études ont continué à évoluer. Depuis 2009, les sagesfemmes interviennent aussi à toutes les étapes de la vie gynécologique et reproductive des femmes en bonne santé. Elles peuvent réaliser en toute autonomie des consultations de contraception et de suivi gynécologique de prévention ${ }^{[13]}$.

La Confédération internationale des sagesfemmes (ICM) a défini la profession de sage-femme et les compétences essentielles qu'elle doit posséder à l'issue de sa formation initiale, que celle-ci soit universitaire ou non universitaire ${ }^{[14,15]}$. Ces compétences s'appuient sur des connaissances et des capacités techniques qui s'acquièrent dans un parcours académique dont le programme et la durée varient dans les Etats membres de l'Union Européenne ${ }^{[16]}$.

Selon la directive 2013/55/UE modifiant la directive 2005/36/CE sur la reconnaissance des qualifications professionnelles, la durée minimale de la formation de sage-femme a été définie soit à trois ans, soit à deux ans en étant subordonnée à la possession d'un titre de formation d'infirmier responsable des soins généraux. Elle peut être définie à 18 mois si elle intervient après une formation de base d'infirmière suivie d'une pratique professionnelle d'un an pour laquelle est délivrée une attestation ${ }^{[17,18]}$. Cette règle s'applique en Suède et au Royaume Uni où, à l'issue de leur formation initiale, les sages-femmes obtiennent une licence en maïeutique (Bachelor of Science in Midwifery). Dans ces deux pays, l'existence d'un master et d'un Doctorat en maïeutique accessible en formation continue (MSc ou PhD in Midwifery) témoigne d'une reconnaissance de la maïeutique comme discipline académique ${ }^{[19-21]}$. Dans ces pays, conformément à la directive européenne, la profession de sage-femme est autonome ${ }^{[22]}$, elle est distincte de celle des infirmières et des médecins ${ }^{[17]}$.

En France, la formation initiale des sagesfemmes françaises présente deux particularités, comparée à celle du reste de l'Europe.

D'une part, la durée de formation est une des plus longues ${ }^{[23]}$. En 2005, dans le cadre de l'harmonisation européenne des formations de l'enseignement supérieur, connue sous le nom de processus de Bologne, a été proposée la création d'une architecture des études supérieures fondée sur trois grades, dite système LMD (Licence ou Bachelor - Master - Doctorat), pour les formations dans le domaine de la santé ${ }^{[24]}$. En 2010, sur la base d'un dossier, déposé au ministère de l'enseignement supérieur et de la recherche par l'Association des sages-femmes enseignantes françaises (ASFEF : ancienne dénomination de la Conférence nationale des enseignants en maïeutique), la France a opté pour une organisation des études de sage-femme en cinq ans. L'arrêté du 19 juillet 2011 relatif au régime des études en vue du diplôme de formation générale en sciences maïeutiques a défini le niveau licence de la formation ${ }^{[25]}$. Ce premier cycle dure trois ans et confère le grade de licence ${ }^{1}$. L'arrêté de 2013 relatif au régime des études en vue du diplôme d'Etat de sage-femme précise les années d'études correspondant au niveau master, soit le deuxième cycle des études en sciences maïeutiques, d'une durée de quatre semestres après le premier cycle ${ }^{[26]}$.

D'autre part, la première année des études de sages-femmes est commune avec celle des médecins, des chirurgiens-dentistes et des pharmaciens ${ }^{[27]}$. En 2002, après dix années d'expérimentation à l'école de sages-femmes de Grenoble, la première année des 
études de sages-femmes, première année du premier cycle des études médicales (PCEM1), est devenue commune avec celle des étudiants de médecine et d'odontologie. L'examen classant après le PCEM1 est alors le mode d'entrée en formation ${ }^{[28]}$. Avec la réforme des études de santé impliquant le travail collaboratif de l'ASFEF et des conférences des doyens de médecine, d'odontologie et de pharmacie, l'année du PCEM1 est transformée en première année commune aux études en santé (PACES). Cette première année de formation des sages-femmes, non reconnue jusque là dans leur cursus, devient la première année du premier cycle des étude en sciences maïeutiques $^{[25,27]}$.

La formation universitaire des sages-femmes françaises, un paradoxe

\section{Une absence d'intégration des écoles de sages- femmes à l'université}

Le code de la santé publique distingue trois grandes catégories de professions de santé : les « professions médicales », les «professions de la pharmacie », les « professions d'auxiliaires médicaux » ${ }^{[29]}$. Les professions médicales comprennent les médecins, les chirurgiens-dentistes et les sagesfemmes ${ }^{[30]}$. Depuis 2010, les étudiants de médecine, d'odontologie, de pharmacie et de maïeutique reçoivent, au cours des deux premiers semestres de leur formation, un enseignement commun ( 50 crédits $\mathrm{ECTS}^{1}$ ) et un enseignement spécifique pour chaque filière $\left(10\right.$ crédits $\left.\operatorname{ECTS}^{1}\right)$. A la fin de cette première année, un examen classant est organisé pour chaque

1. L'European Credit Transfer and Accumulation System (ECTS). L'ECTS repose sur le principe selon lequel le travail à fournir par un étudiant à temps plein pendant une année universitaire correspond à 60 ECTS. La charge de travail d'un étudiant inscrit dans un programme d'études à plein temps en Europe étant, dans la plupart des cas, d'une durée d'environ 1500-1800 heures/an, la valeur d'un crédit représente dans ces cas environ 25 à 30 heures de travail (cours, séminaires, travail personnel, préparation des examens..). (Extrait d'Education et Formation, Commission Européenne Socrates, ECTS). filière afin que l'étudiant s'oriente en fonction de son projet professionnel selon la discipline choisie ${ }^{[31]}$. A l'issue des cinq années de formation, le diplôme d'Etat de sage-femme confère de plein droit le grade de master. Le diplôme d'Etat et le grade sont délivrés par les universités françaises habilitées ${ }^{[25-26]}$.

Le principe de la délivrance aux titulaires d'un diplôme d'Etat relevant du domaine de la santé, d'un grade équivalent au titre universitaire, comme pour d'autres formations (architectes,...), a été retenu pour plusieurs raisons ${ }^{[29]}$ :

- en France une même formation ne peut pas conduire à la délivrance de deux diplômes différents, ce qui serait par exemple le cas avec une licence d'infirmier et un diplôme d'Etat d'infirmier (diplôme d'exercice) ;

- les diplômes de licence et master sont des diplômes obtenus à partir d'un contenu de formation qui est sous la seule responsabilité des universités, dans le cadre de leur autonomie pédagogique ; or les formations des professions de santé doivent conserver un cadrage national, comme l'exigent les diplômes d'exercice des professions réglementées ;

- l'accès aux formations médicales et à certaines études paramédicales est soumis à un numerus clausus ou un quota.

La première année des études de sages-femmes se déroule à l'université ${ }^{[29]}$ mais, contrairement aux études de médecine, d'odontologie ou de pharmacie, les quatre années suivantes sont organisées au sein d'écoles agrées par les régions et gérées par les hôpitaux et non par les universités ${ }^{[32]}$. Pour autant, les étudiants sages-femmes sont inscrits à l'université tout au long de leur cursus.

Depuis la loi du 21 juillet 2009, les écoles peuvent être intégrées à l'université ${ }^{[13]}$. A ce jour, une seule école de sages-femmes, l'école universitaire de Marseille, a pleinement intégré l'université sous la forme d'une composante autonome ${ }^{[33]}$.

Enfin, alors que le code de l'éducation précise les dispositions propres aux unités de formation et de recherche (UFR) de médecine, pharmacie, odontologie et maïeutique, seules les études de médecine, d'odontologie et de pharmacie sont dispensées au sein d'UFR, composantes des universités ${ }^{[34]}$. 


\section{Une absence de statut universitaire pour les enseignantes sages-femmes}

Les sages-femmes enseignantes sont, en grande majorité, employées par les hôpitaux. Jusqu'en 2011, pour devenir enseignante, il était obligatoire d'être titulaire du diplôme de cadre sage-femme délivré, après une formation de neuf mois, dans l'unique école hospitalière française, située à Dijon ${ }^{[35]}$. Le diplôme de cadre sage-femme, de nature non universitaire, a été remplacé par un diplôme de master en santé publique, spécialité «périnatalité, management et pédagogie » (master PMP), délivré après deux années de formation, par l'Université de Bourgogne. Cette formation s'adresse aux professionnels de santé en situation de responsabilité dans le champ de la périnatalité (médecins, puéricultrices ou sages-femmes). En deuxième année, les étudiants optent pour un des trois parcours proposés : management, pédagogie ou recherche. Comme antérieurement pour le diplôme de cadre, ce diplôme de master, quel que que soit le parcours suivi, permet l'accès à la fonction de sagefemme enseignante ${ }^{2}$ ou de sage-femme assurant des fonctions managériales dans des maternités.

L'accès à la fonction de directrice ${ }^{2}$ est possible pour les sages-femmes titulaires du diplôme de sagefemme cadre de Dijon ou du master PMP suite à un concours sur titres et sur épreuves organisé par l'établissement hospitalier dont l'école dépend. Seule la réussite au concours permet l'accès au grade de sagefemme directrice ${ }^{[36]}$.

Il n'existe donc pas d'exigence de formation de nature universitaire, ni d'exigence de formation en pédagogie ou en recherche pour le corps enseignant sage-femme alors qu'un grade de master est délivré aux étudiants sages-femmes.

Les sages-femmes en Suède ou au Royaume Uni, contrairement à la France, ne sont pas considérées

2. En décembre 2014, le statut hospitalier des sages-femmes françaises a changé, supprimant la notion de sage-femme cadre, le statut des directeurs d'école de sages-femmes ainsi que le concours de directrice et considérant les sages-femmes selon deux grades : le grade 1 et le grade 2 . Ce dernier comprend les sages-femmes cadres. Un grade de coordonnateur en maïeutique a été créé pour les directrices. Nous n'avons pas tenu compte de ces changements dans notre étude car l'enquête a été réalisée avant la parution des textes. comme profession médicale. Pour autant elles disposent d'un statut universitaire et les structures de formation sont intégrées au sein des universités comprenant des composantes en santé ${ }^{\text {[37,38] }}$. L'enseignement en maïeutique, fondé sur des données probantes issues de recherches conduites par des équipes multidisciplinaires (sages-femmes, pharmaciens, infirmières, médecins, épidémiologistes, sociologue, psychologue, anthropologues, etc.) est dispensé essentiellement par les sages-femmes ${ }^{[37,39]}$.

La Conférence nationale des enseignants en maïeutique (CNEMa), est une association régie par la loi du 1er juillet 1901, représentative de l'ensemble des écoles comprenant le corps enseignant sagefemme. A l'instar des conférences des doyens de médecine, d'odontologie ou de pharmacie, elle participe à la réflexion sur les formations de santé et la présidente siège au Comité de l'Observatoire national de la démographie des professions de santé (ONDPS) ainsi qu'à la Commission nationale des études de maïeutique, médecine, odontologie et pharmacie (CNEMMOP) ${ }^{[40,41]}$. La CNEMa constitue une force de propositions et de décisions politiques en matière de formation, de soins et de recherche dans le domaine de la maïeutique, auprès des autorités ministérielles et des instances ordinales nationales. Elle entretient des liens étroits avec les organisations professionnelles de sages-femmes françaises et les sages-femmes des autres pays en participant, notamment, aux réunions et congrès de l'ICM (International Confederation of Midwives) et de l'EMA (European Midwives Association). En 2008, dans son rapport, la mission interministérielle sur « La reconnaissance des diplômes d'Etat professionnels paramédicaux et de sages-femmes par référence au schéma universitaire européen LicenceMaster-Doctorat » déclarait indispensable une évolution des méthodes et du contenu de l'enseignement qui était dispensé aux sages-femmes, car « les méthodes pédagogiques sont même parfois jugées archaïques et infantilisantes, les contenus décalés par rapport aux avancées médicales » ${ }^{[35]}$. Dans le cadre de la réflexion qui a conduit à la réforme des études de sages-femmes, la CNEMa a été chargée d'émettre des avis et des propositions relatives aux questions pédagogiques spécifiques concernant l'évolution des 
études de maïeutique. Ainsi, le programme de formation a été mis en adéquation avec les compétences des sages-femmes et répond aux besoins sociétaux dans le champ de la santé périnatale et génésique. La CNEMa a conduit les travaux aboutissant à la rédaction du nouveau référentiel de formation pour le diplôme d'Etat de sage-femme ${ }^{[42]}$.

Son rôle d'expertise et d'instance représentative de la formation des sages-femmes françaises auprès des tutelles ministérielles a mené la CNEMa à recenser le nombre de sages-femmes enseignantes possédant un diplôme universitaire.

L'étude s'est inscrite dans le cadre des demandes des sages-femmes françaises pour une meilleure reconnaissance de leur profession et de leur rôle auprès des femmes et nouveau-nés ${ }^{[43]}$ ainsi que pour une évolution du statut des sages-femmes. Trois sollicitations spécifiques concernaient l'enseignement :

- formation des sages-femmes dans des structures universitaires respectant l'autonomie de la filière maïeutique ;

- statut universitaire pour les sages-femmes enseignantes garantissant une gestion autonome tout en leur permettant de conserver l'indispensable activité éducative et clinique indissociables ainsi que le développement de la recherche en maïeutique nécessaire à l'amélioration la santé des femmes et des nouveau-nés ;

- création d'une section maïeutique au Conseil national des universités (CNU) qui reconnaisse cette discipline académique.

\section{Objectifs de l'étude}

L'objectif principal de l'étude était de connaître les diplômes universitaires détenus par les sagesfemmes enseignantes et directrices. L'objectif secondaire était de rapporter des informations utiles pour orienter les décisions concernant l'évolution du statut du corps enseignant sage-femme.

\section{Méthodes}

\section{Schéma d'étude}

Il s'agit d'une enquête par questionnaire autoadministré, structuré, à questions fermées auprès de l'échantillon exhaustif des 241 sages-femmes enseignantes et sages-femmes directrices des 35 écoles de sages-femmes françaises.

\section{Population étudiée}

Toutes les sages-femmes enseignantes et directrices exerçant dans les structures de formation en maïeutique étaient éligibles.

\section{Critères d'inclusion}

Toutes les sages-femmes enseignantes y compris celles en congé de formation ont été incluses.

\section{Critères d'exclusion}

Les sages-femmes intervenant ponctuellement dans les écoles, les sages-femmes enseignantes retraitées, en disponibilité ou en détachement pour exercer en service de Protection Maternelle et Infantile ou en exercice libéral ont été exclues.

\section{Organisation de l'étude}

Deux membres du bureau de la CNEMa ont été chargés de la coordination de l'enquête (envoi des questionnaires, enregistrement des réponses et relances).

Le transfert du questionnaire vers le corps enseignant sage-femme a été confié aux 35 sages-femmes directrices. Un message électronique, comprenant une lettre d'information et le questionnaire présenté sous forme de tableau Excel, a été envoyé à chaque sage-femme directrice. Cette dernière était chargée de diffuser l'information sur l'enquête auprès de l'équipe pédagogique et de regrouper l'ensemble des réponses.

Le caractère exhaustif de l'enquête a pu être contrôlé à partir des fiches d'information que communiquent régulièrement les sages-femmes directrices. En effet, que ce soit spontanément à l'ensemble des écoles de sages-femmes ou sur demande de la CNEMa, chaque responsable transmet régulièrement l'organigramme de l'école précisant le nombre et le 
nom de chaque membre de l'équipe pédagogique. La dernière mise à jour des organigrammes dénombrait 241 sages-femmes enseignantes et directrices en 2014.

Le questionnaire a été distribué le 7 novembre 2014. Les équipes pédagogiques disposaient de quinze jours pour y répondre. Une relance par messagerie électronique a été nécessaire vers les écoles qui n' avaient pas répondu. Afin d'obtenir les réponses de trois écoles non répondantes, deux échanges téléphoniques ainsi qu'une visioconférence ont été nécessaires. Ces différentes démarches ont permis d'obtenir les réponses de la totalité des sages-femmes enseignantes.

\section{Elaboration du questionnaire}

L'objectif étant d'avoir une description la plus complète possible des diplômes détenus par les sages-femmes enseignantes et directrices des écoles de sages-femmes françaises, il s'agissait d'un questionnaire unique, très simple, comprenant neuf questions. L'outil de recueil n'a donc pas été rédigé à partir d'une revue ciblée de la littérature.

Il était demandé à chaque répondant de fournir les informations suivantes :

- situation géographique de la structure de formation

- fonction (sage-femme enseignante ou sage-femme directrice),

- grade selon ceux définis par la fonction publique hospitalière ${ }^{3}$ (sage-femme classe normale ou classe supérieure, sage-femme cadre ou cadre supérieure, sage-femme directrice $)^{1}$,

- date de naissance,

- quotité de travail (temps plein ou heures de travail hebdomadaire pour les enseignantes à temps partiel),

- titulaire ou non du diplôme de sage-femme cadre de Dijon,

- grade du diplôme universitaire le plus élevé obtenu (licence, master dont le master PMP ou doctorat),

- nombre de diplômes universitaires (DU) ou interuniversitaires (DIU) validés,

- diplômes universitaires en cours.
Le questionnaire a été testé auprès de trois enseignantes et une directrice. Aucune difficulté de compréhension n'a été relevée et aucun commentaire n'a été exprimé. Aucune modification n'ayant été apportée après cette phase test, les quatre questionnaires tests ont été inclus dans l'enquête.

Lorsque des données aberrantes ont été identifiées, les directrices des écoles ont été recontactées pour vérification et, si nécessaire, correction.

\section{Analyse des résultats du questionnaire}

Les données, reprises sur fichiers Excel, ont été transférées sur un fichier du Logiciel R, version 2.15.2. Une analyse statistique descriptive a été entreprise par une sage-femme chercheure et vérifiée par le premier auteur de l'article. L'âge, variable continue, a été décrite, dans un premier temps, en moyenne et écart-type puis, dans un deuxième temps, a été discrétisée en amplitude d'intervalle de cinq ans et analysé par catégorie. Toutes les variables qualitatives ont été décrites en effectifs et pourcentages.

\section{Résultats}

Les équipes pédagogiques des 35 écoles ont renvoyé leurs réponses via les directrices. Une des directrices assumait la responsabilité de la direction de deux écoles; les analyses ont donc porté sur un total de 241 répondants comprenant 34 directrices et 207 sages-femmes enseignantes.

\section{Age des membres des équipes pédagogiques}

Deux répondants n'ont pas renseigné leur âge. Les âges rapportés variaient entre 24 et 71 ans, avec une moyenne de 47 ans +/-.8,3. La fourchette d'âge s'étendait de 40 à 71 ans (moyenne 53 ans +/-.5,6) pour les directrices et de 24 à 63 ans, (moyenne 46 ans $+/-.8,3$ ) pour les enseignantes. L'âge a été analysé par catégories. Les fréquences cumulées ont permis de mettre en évidence que $199 / 239$, soit $83,2 \%$ des 
Tableau I. Diplôme le plus élevé (hors DU/DIU*) des sages-femmes enseignantes et directrices.

\begin{tabular}{|l|c|c|c|}
\cline { 2 - 4 } \multicolumn{1}{c|}{} & $\begin{array}{c}\text { Directrices } \\
n(\%)\end{array}$ & $\begin{array}{c}\text { Enseignantes } \\
n(\%)\end{array}$ & $\begin{array}{c}\text { Toutes } \\
n(\%)\end{array}$ \\
\hline Diplôme d'Etat & $0(0)$ & $52(25)$ & $52(21,6)$ \\
\hline Diplôme cadre & $16(47)$ & $66(32)$ & $82(34)$ \\
\hline Master & $16(47)$ & $83(40)$ & $99(41,1)$ \\
\hline Master PMP & $1(3)$ & $5(2,5)$ & $6(2,5)$ \\
\hline Doctorat & $1(3)$ & $1(0,5)$ & $2(0,8)$ \\
\hline Total & $34(100)$ & $207(100)$ & $241(100)$ \\
\hline
\end{tabular}

*DU/DIU (diplôme d'université ou interuniversitaire).

enseignantes actuelles étaient âgées de 40 ans ou plus, et $98 / 239(41 \%)$ avaient plus de 50 ans.

\section{Quotité de temps de travail des équipes pédagogiques}

Tous les répondants, sauf un, ont précisé leur quotité de temps de travail. La grande majorité (196/240, $81,7 \%)$ travaille à temps plein. Une sage-femme enseignante suivant un programme de master n'exerçait aucune activité à l'école au moment de l'enquête. La quasi totalité des sages-femmes directrices travaillait à temps plein, une travaillant à $80 \%$ et une autre à $50 \%$. Cette dernière était une directrice âgée de 71 ans.

\section{Diplôme le plus élevé}

Au total (tableau I), presque la moitié des sagesfemmes enseignantes ou directrices possédaient un diplôme de master ou de doctorat (107/241, 44,4 \%).

Trente-deux des 34 sages-femmes directrices avaient obtenu le diplôme de sage-femme cadre ${ }^{1}$, celui-ci étant le diplôme le plus élevé pour 16 d'entre elles (47\%). Parmi les 18 restantes, 17 avaient un master, dont une le master PMP de l'Université de Bourgogne. Une sage-femme directrice possédant le diplôme cadre était aussi titulaire d'un doctorat en sciences de l'éducation. Au total, neuf directrices sur $34(26,4 \%)$ n'avaient pas le grade de directrice mais assuraient les fonctions de direction.
Parmi les 207 sages-femmes enseignantes, 66 (32\%) avaient le diplôme cadre et $88(42,5 \%)$ un master, dont cinq celui de l'Université de Bourgogne. Une sage-femme enseignante $(0,5 \%)$ était titulaire d'un doctorat en épidémiologie. Enfin, 52 sagesfemmes enseignantes ( $25 \%$ ) avaient, pour diplôme le plus élevé, le diplôme de sage-femme. Parmi elles, huit étaient titulaires d'un diplôme d'université (DU ou DIU).

\section{Diplôme en cours}

Vingt-quatre des 34 sages-femmes directrices et 156 des 207 sages-femmes enseignantes ont répondu à la question portant sur les formations en cours (tableau II). Deux directrices et 18 enseignantes étaient en cours de doctorat dans des disciplines variées (sociologie, santé publique/épidémiologie, philosophie, sciences de l'éducation). Cinq directrices et 32 enseignantes suivaient un programme de master. Parmi les 52 sages-femmes enseignantes qui avaient pour diplôme le plus élevé, le diplôme de sage-femme, 19 suivaient une formation de niveau master. Enfin, 119 sages-femmes enseignantes et 30 sages-femmes directrices, suivaient ou avaient suivi entre un et quatre DU ou DIU.

\section{Discussion}

Cette enquête entreprise par la CNEMa, avait pour objectif principal de recueillir des informations 
Tableau II. Diplôme en cours (y compris DU/DIU*) des sages-femmes enseignantes et directrices

\begin{tabular}{|c|c|c|c|}
\hline & $\begin{array}{c}\text { Directrices } \\
n(\%)\end{array}$ & $\begin{array}{c}\text { Enseignantes } \\
n(\%)\end{array}$ & $\begin{array}{l}\text { Toutes } \\
n(\%)\end{array}$ \\
\hline $\begin{array}{l}\text { Aucun } \\
\text { Licence }\end{array}$ & $\begin{array}{c}16(66,7) \\
0(0)\end{array}$ & $\begin{array}{c}91(58,3) \\
1(0,6)\end{array}$ & $\begin{array}{c}107(59,5) \\
1(0,6)\end{array}$ \\
\hline Master & $4(16,7)$ & $27(17,3)$ & $31(17,2)$ \\
\hline Master PMP & $1(4,1)$ & $5(3,2)$ & $6(3,3)$ \\
\hline Doctorat & $2(8,3)$ & $18(11,6)$ & $20(11,1)$ \\
\hline $\begin{array}{l}\text { DU/DIU } \\
\text { Total }\end{array}$ & $\begin{array}{c}1(4,2) \\
24(100)\end{array}$ & $\begin{array}{c}14(9) \\
156(100)\end{array}$ & $\begin{array}{c}15(8,3) \\
180(100)\end{array}$ \\
\hline
\end{tabular}

* DU/DIU (diplôme d'université ou interuniversitaire).

$¥$ Question non renseignée pour 34 sages-femmes directrices et 51 sages-femmes enseignantes.

sur les diplômes universitaires détenus par les 241 sages-femmes enseignantes et directrices exerçant dans les 35 écoles de sages-femmes françaises.

Le recueil des réponses a été effectué selon une méthode simple de collecte de données afin d'obtenir le plus grand nombre possible de réponses des sagesfemmes enseignantes et directrices sur une très courte période. Un taux de réponses de $100 \%$ a permis d'établir une représentation très précise de la situation académique des sages-femmes enseignantes en France.

Etabli sous forme de tableur, le questionnaire comportait seulement neuf questions. Quoi qu'il en soit, la simplicité de l'enquête a permis le recueil des réponses de toutes les enseignantes et directrices en France métropolitaine et Outre-mer. Ceci a sans doute pu être réalisé, également, grâce aux liens professionnels entretenus entre les écoles, les enseignantes et directrices et la CNEMa.

Il s'agit de la première étude qui décrit l'âge et les diplômes détenus par les équipes pédagogiques des écoles de sages-femmes. L'analyse de l'âge affiche une moyenne de 47 ans, ce qui est comparable à la moyenne d'âge de 46 ans des médecins, dentistes et pharmaciens qui enseignent à l'université ${ }^{44]}$. Elle montre que plus de $80 \%$ des sages-femmes enseignantes et directrices sont âgées de 40 ans et plus, ce qui est cohérent compte tenu de l'expérience clinique et pédagogique nécessaire pour ces professionnelles et de la formation continue quasi obligatoire pour accéder à la fonction. Dix-neuf $(9,1 \%)$ enseignantes ont moins de 35 ans, dont six (2,8 \%) moins de 29 ans. Cette dernière tranche d'âge soulève la question de l'expérience professionnelle et de la préparation de ces enseignantes au rôle éducatif, mais également au rôle de soutien dont elles disposent au sein de l'équipe enseignante. Le rajeunissement des équipes pédagogiques pourrait s'accentuer avec la réforme des études de sages-femmes françaises. En effet, il est maintenant possible d'accéder à une deuxième année de master à orientation recherche puis à un doctorat immédiatement après l'obtention du diplôme d'Etat pour les étudiants qui auront suivi un parcours recherche pendant leur formation initiale. Ce rajeunissement des sages-femmes titulaires de diplôme de $2^{\text {ème }}$ ou de $3^{\text {ème }}$ cycle, complémentaire au diplôme d'Etat de sage-femme, amènera à considérer, comme c'est le cas dans les filières de médecine, d'odontologie ou de pharmacie, l'attribution des postes des sages-femmes enseignantes, sur des critères autres que l'expérience clinique, l'âge et/ou l'ancienneté des candidates ${ }^{[44]}$. Le projet professionnel, la qualité des candidats, leur potentiel en termes d'enseignement et de recherche, et leurs publications scientifiques, seront également des indicateurs importants à prendre en considération ${ }^{[44]}$. En effet, l'inscription de la maïeutique dans les standards de la littérature internationale, au travers des publications de sagesfemmes dans des revues internationales à comité de lecture, serait garant de la qualité des travaux et de l'information transmise aux étudiants. De plus, une promotion à un poste universitaire est soumise à des 
prérequis en termes de publications. Selon Joyce Thompson, les qualifications nécessaires pour assurer les fonctions de sage-femme enseignante comprennent une formation officielle en pédagogie, la compétence en pratique clinique, l'aptitude à penser de manière critique et à enseigner la pensée critique, et un engagement à un développement professionnel continu tout au long de la vie pour eux-mêmes et pour leurs étudiants ${ }^{[7]}$. Pour cette même sage-femme et pour l'ICM, l'approfondissement des compétences et la confiance indispensable avant d'assumer la responsabilité de former des étudiants sages-femmes nécessitent au moins deux à cinq années de pratique clinique dans des domaines d'exercice variés ${ }^{[7,45]}$. Selon Metté et al, pour devenir enseignants, la compétence médicale et l'expérience suffisent pour le transfert d'informations. Par contre, pour aider l'étudiant à utiliser des connaissances en contexte d'incertitude, à raisonner et à prendre des décisions pour résoudre des problèmes complexes, dans un contexte émotionnel fort et en tenant compte de conflits éthiques et culturels, d'autres compétences sont nécessaires ${ }^{[46]}$. Parmi ceux-ci, une formation approfondie à la démarche de recherche semble essentielle.

La recherche en santé et la clinique sont intimement liées et savoir extraire de la littérature scientifique les éléments qui permettent d'en apprécier la pertinence, la portée et les limites sont les marques du bon praticien ${ }^{[47]}$. Les résultats des études internationales amènent à une constante remise en question des pratiques des sages-femmes. Ces dernières, tout au long de leur vie professionnelle doivent approfondir leurs connaissances et parfois même remettre en cause des dogmes bien établis qu'elles ont ellesmêmes adoptés et appliqués. Certains, tels que l'enregistrement systématique du rythme cardiaque fœetal pendant le travail, l'amniotomie et la perfusion d'ocytocine pour accélérer le travail, dans un contexte de grossesse à bas risque et de travail physiologique sont inappropriés au regard des nouvelles connaissances fondées sur les preuves ${ }^{[5,8,9]}$. Les sagesfemmes britanniques et suédoises, enseignantes et cliniciennes ont intégré depuis longtemps la nécessité d'évaluer leurs pratiques, de mener des études sur la prise en charge qu'elles proposent aux femmes, d'appuyer leur exercice professionnel sur les résultats des travaux menés de façon rigoureuse et sur l'Evidence-Based Practice ou sur l'Evidence Based Medecine ou Midwifery. Titulaires de PhD (Doctorat d'Université) ou en cours de formation doctorale, premiers auteurs d'articles scientifiques, elles utilisent, les revues académiques mondialement connues des experts et sociétés savantes, pour publier les résultats de leurs travaux de recherche et apporter des connaissances nouvelles dans leur champ d'exercice. Ces connaissances sont diffusées aux sages-femmes, aux étudiantes sages-femmes et à tous les professionnels de santé qui gravitent autour de la femme enceinte et du nouveau-né $[1,3,4,8,48]$.

Les recherches menées par des sages-femmes pour évaluer des stratégies ou dispositifs pédagogiques développés pour favoriser 1'acquisition des compétences par les étudiants sont également très développés en Suède et au Royaume Uni ${ }^{[19,37,49]}$. Les modalités pédagogiques et didactiques recommandées dans le nouveau programme des études de sagesfemmes françaises, et choisies par les équipes enseignantes pour faciliter l'apprentissages des étudiants sages-femmes et développer leur compétences, demandent aussi à être évaluées : approche par compétences, pratique réflexive à l'aide de présentations et études de cas, analyses de pratiques, ateliers réflexifs sur la base de situations réelles ou fictives, ateliers de simulations cliniques et de retours d'expérience sur les périodes de formation clinique, cours magistraux, enseignements dirigés et pratiques mutualisées, pratiques d'encadrement ou d'évaluations formatives et personnalisées. La conduite de tels projets de recherche peut être facilitée par la présence de sages-femmes enseignantes chercheures au sein des équipes pédagogiques.

L'arrêté de 2009 relatif aux modalités d'agrément des écoles de sages-femmes françaises précise dans son article 2 que les écoles de sages-femmes agréées ont pour mission [...] le développement d'activité de recherche d'intérêt professionnel ${ }^{[32]}$. Le nouveau programme des études recommande que la formation des sages-femmes s'appuie sur une approche intégrant des données factuelles (données probantes) au moyen de présentations et de revues de la littérature, d'analyses d'articles scientifiques dans le domaine de la maïeutique, de cours dispensés par des chercheurs 
ou doctorants ou construits sur la base de résultats de recherches et de bibliographies actualisées ${ }^{[26]}$ Les équipes pédagogiques des écoles de sages-femmes ont le devoir de développer chez les étudiants le sens critique qui doit transparaître dans leurs pratiques professionnelles et leurs travaux de recherche, de former des professionnels compétents qui exerceront en s'appuyant sur des preuves scientifiques ${ }^{[47]}$. L'atteinte de ces objectifs serait facilitée par un enseignement à la démarche de recherche dispensé par un corps enseignant sage-femme qui dispose d'un diplôme de niveau I (diplôme de master ou doctorat).

En matière de diplômes universitaires, presque la moitié des enseignantes et directrices est titulaire d'un diplôme de master (107/241, 44,4 \%) et 37 sont en cours d'acquisition d'un tel diplôme ( 5 directrices et 32 sages-femmes enseignantes). Ces résultats témoignent de la volonté des sages-femmes enseignantes et directrices d'obtenir des titres universitaires leur permettant d'être au moins à un niveau universitaire similaire aux étudiants qu'elles forment. Il montre aussi le paradoxe entre l'existence d'étudiants dont la formation mène au grade de master et leur encadrement pédagogique qui, pour trente-trois (16\%) sages-femmes enseignantes, n'a pas de diplôme complémentaire (diplôme acquis ou en cours d'acquisition). De plus, neuf directrices sur 34 $(26,4 \%)$ n'avaient pas le grade de directrice mais assuraient les fonctions de direction ${ }^{3}$. Ces chiffres soulèvent deux questions : celle relative au processus de recrutement des sages-femmes enseignantes titulaires du diplôme de cadre sage-femme ou du master PMP qui n'a pas toujours été pris en compte, et celle relative à la direction des écoles sans condition de réussite à un concours ou d'acquisition de diplôme de nature universitaire. Pour les sages-femmes non titulaires des diplômes requis pour exercer ces fonctions, une période transitoire pourrait être compréhensible avant de s'engager dans la formation universitaire, mais au delà d'une certaines durée il aurait été souhaitable que ces personnes se mettent en conformité avec la réglementation.

Le diplôme de master ouvre la voie au doctorat et les résultats de l'étude montrent qu'une enseignante et une directrice possèdent une thèse d'Université et que 20 sont doctorantes (22/241, $9 \%$ ). En 2010, parmi les
24309 sages-femmes inscrites au tableau de l'ordre des sages-femmes en France, le Conseil de l'Ordre des sages-femmes déclarait connaître sept sages-femmes possédant un doctorat ${ }^{[50]}$. En début d'année 2014, à la demande du Ministère de l'Enseignement Supérieur et de la Recherche, une enquête réalisée par la CNEMa et à laquelle avaient répondu 1752 sages-femmes, révélait l'existence de 44 sages-femmes chercheurs (3\% des répondants) dont 11 étaient titulaires d'un doctorat. Dix-neuf de ces 44 sages-femmes chercheurs appartenaient au corps enseignant (enquête non publiée). Cette évolution académique des niveaux de diplômes des sages-femmes enseignantes répond aux recommandations portées par l'IGAS et l'IGAENR : «S'agissant de la formation, la transposition du dispositif LMD peut difficilement s'imaginer sans que les enseignantes des écoles, même s'ils ne sont pas les seuls intervenants, ne soient recrutés dans les mêmes conditions que les enseignants de l'enseignement supérieur ${ }^{[35]}$.

Le nombre de sages-femmes détentrices d'un doctorat reste toutefois remarquablement faible comparé à celui du Royaume Uni ou de la Suède ${ }^{[51]}$. En 2014, le College of Nursing and Midwifery au Royaume Uni a dressé une liste de 24 infirmières et sages-femmes professeurs d'Université, dont neuf étaient présidentes d'université ${ }^{[52]}$. Marlène Sinclair, sage-femme et présidente de la Chaire de recherche en maïeutique à l'Université d'Ulster (Irlande) rapportait, il y a presque dix ans, que le nombre de doctorants dans chaque université des quatre pays du Royaume Uni variait entre zéro et sept avec une moyenne de deux par université ${ }^{[20]}$. Son enquête auprès de professeurs de maïeutique faisait état d'environ 50 sages-femmes en formation doctorale. Bien que ces chiffres ne soient pas récents, le nombre de sages-femmes doctorantes, qui ne peut qu'avoir évolué en huit ans, est témoin d'une culture de la recherche très marquée chez les sages-femmes britanniques.

La France accuse un retard dans le nombre de sages-femmes titulaires de diplômes universitaires de 3ème cycle par rapport aux deux pays européens cités en comparaison ${ }^{[51]}$. Ce retard académique est également manifeste lorsque l'on compare le nombre sages-femmes françaises qui dirigent des travaux de 
recherche, par rapport à d'autre pays, qu'ils soient européens ou non, tels que le Canada, la Suisse ou la Grèce ${ }^{[53-56]}$. Plusieurs universités préparant à la profession de sages-femmes, comme celles de Central Lancashire (School of Community Health and Midwifery) ${ }^{[57]}$, ou de King's College à Londres (The university King's College London) ${ }^{[58]}$, disposent de laboratoire de recherche dans lesquels des sagesfemmes chercheures proposent et mènent des travaux relevant de leur champ de compétence.

Une seule sage-femme enseignante a déclaré avoir pris un congé pour suivre une formation de master. La grande majorité ( $82 \%$ ) de la population étudiée a indiqué une quotité de travail à temps complet en même temps qu'elle poursuivait un programme de master ou doctorat. Le statut hospitalier des sagesfemmes ne bénéficie pas des mêmes dispositions que celui des personnels universitaires en termes de recherche et de formation continue (financement des formations doctorales, temps dédié, reconnaissance des activités de recherche ou de lecture d'articles scientifiques, soutien à la publication etc.). Ceci constitue sans doute un obstacle à l'acquisition des masters ou doctorat pour les sages-femmes et au développement de la recherche clinique ou en pédagogie du champ de la maïeutique.

L'histoire française montre que la maternité est avant tout abordée de manière médicale par les médecins et non comme un phénomène sociétal, de la sphère privée, intime et familiale ${ }^{[10]}$. De plus, l'histoire de la surveillance de la grossesse et de l'accouchement s'est inscrite dans le cadre d'une lutte pour l'exercice de la profession entre les accoucheurs, essentiellement des hommes, et les sagesfemmes ${ }^{[12]}$. Avant la réforme LMD, la formation des sages-femmes qui était en France sous la responsabilité et le contrôle des médecins chargés d'organiser les cours et de délivrer l'enseignement théorique, a conduit à un enseignement fondé sur la conception d'une médecine technique, de pointe et curative qui prévaut toujours dans les centres hospitaliers universitaires $^{[12]}$. Enfin, jusqu'en 2010 , les écoles étaient certes dirigées par des sages-femmes directrices, mais sous la responsabilité de directeurs techniques, obstétriciens, professeur des universités - praticien hospitalier (PU-PH) ${ }^{[59]}$. Cette situation singulière des sages-femmes françaises explique sans doute le peu de recherche menée, encore aujourd'hui, dans leur pays, sur la déambulation pendant le travail et les positions d'accouchement pour favoriser la physiologie de la naissance, les alternatives à la péridurale pour soulager la douleur des contractions, la précarité sociale des femmes enceintes pour une meilleure prise en charge ou encore sur les conséquences psychologiques et économiques de la surmédicalisation de la grossesse et de l'accouchement, etc. Le choix des thèmes des mémoires des étudiants sages-femmes traitent souvent de pathologies, hors du champ de compétences des sages-femmes ${ }^{[60]}$. Le faible nombre d'enseignantes sages-femmes titulaires de doctorat dans les écoles françaises, peut expliquer le choix de l'orientation des thèmes des mémoires. Les principaux objectifs du mémoire sont d'initier les étudiants à la démarche scientifique, de former des professionnels capables d'adopter une attitude réflexive par rapport à leur pratique et de la fonder sur des données probantes afin de contribuer à l'amélioration de la qualité des soins ainsi qu'à l'évolution de la profession ${ }^{[61]}$. Ces objectifs s'accordent avec les compétences essentielles attendues des sages-femmes ${ }^{[62]}$ et avec les règles déontologiques de la profession ${ }^{[63]}$. Promouvoir la culture de la recherche chez les étudiants sages-femmes nécessite des sages-femmes enseignantes, modèles de recherche auxquels les étudiants peuvent s'identifier, qui utilisent des méthodes d'enseignement et d'apprentissage régulièrement mises à jour sur la base des dernières données de la science. La direction d'un mémoire exige des compétences minimales en matière de lecture critique de la littérature scientifique et de méthode de recherche.

Alors qu'aujourd'hui, les résultats convergent pour inciter les pays à revenus élevés à mener les actions en faveur d'une prise en charge de la grossesse et de l'accouchement moins interventionniste, davantage centrée sur les besoins des femmes ${ }^{[64]}$, la création d'un corps enseignant sage-femme chercheur trouve ici tous son sens. Formés à la recherche, ces acteurs privilégiés auprès des femmes en bonne santé pour leur suivi gynécologique, leur grossesse ou leur accouchement, répondraient alors aux préconisations des auteurs du Lancet ${ }^{[5,8,9]}$. pour participer 
à l'amélioration de la santé maternelle et néonatale, mais aussi pour contribuer au développement personnel, professionnel et académique de l'étudiant sagefemme et de ses pairs ${ }^{[65,66]}$.

A la lumière de nos résultats, qui n'ont pas pris en compte le changement de statut hospitalier de sage-femme intervenu en $2014^{3}$, une nouvelle enquête semble nécessaire. Il est toutefois important de préciser que ce nouveau statut, comme le précédent, ne permet pas une double appartenance « hospitalo-universitaire $\gg$ des sages-femmes enseignantes. Pourtant l'exercice hospitalo-universitaire, qui repose sur les missions de soin, d'enseignement et de recherche, apparaît comme le garant du transfert de l'excellence universitaire vers les soins apportés aux femmes et aux nouveau-nés. Il est permis aux deux autres professions médicales, il n'est pas accessible au corps enseignant des sages-femmes. L'évolution du statut des sages-femmes enseignantes vers un corps d'enseignants chercheurs en maïeutique comprenant la possibilité d'exercer cette triple mission, permettrait sans doute, aux femmes et aux couples, de bénéficier des soins adaptées à leur niveau de risque et à leur choix.

Aux données quantitatives exposées dans notre étude, il serait justifié d' ajouter des données plus qualitatives dont celles relatives aux modalités pratiques de déroulement des formations universitaires suivies par les enseignantes sages-femmes (mode de prise en charge financière, formation sur le temps de travail ou non, décharge des missions d'enseignement, organisation interne des équipes pédagogiques) et celles portant sur les activités pédagogiques et de recherche des sages-femmes enseignantes.

\section{Conclusion}

Cette étude, simple aussi bien dans sa conception que dans ses objectifs, a permis de décrire le corps enseignant des sages-femmes qui, ces dernières années, s'est inscrit dans des parcours de formation universitaire de niveau master et doctorat. Une comparaison avec les titres universitaires détenus par des sages-femmes au Royaume Uni et en Suède a permis de soulever un certain nombre de questions dont celle de la recherche en maïeutique quasi inexistante en
France. Seulement deux sages-femmes enseignantes étaient détentrices d'un doctorat en 2014. Cette enquête a aussi montré l'absence de formation spécifique requise pour exercer la fonction de sage-femme enseignante ou directrice. Les sages-femmes qui exercent en France dans les écoles de sages-femmes sont de plus en plus nombreuses à détenir un diplôme de master. Les titulaires de thèses d'université sont encore en nombre insuffisant dans les écoles de sagesfemmes bien que le chiffre de vingt doctorantes soit encourageant.

Cet état des lieux des diplômes détenus par les sages-femmes enseignantes en France et les données issues du Royaume Uni ou de la Suède, offrent l'opportunité de débats avec les pouvoirs publics afin de favoriser l'évolution du rôle des sages-femmes sur le territoire français, notamment dans le cadre de leur lieu de formation et de la recherche en maïeutique.

\section{Contributions}

Christine Morin et Marie-Christine Leymarie ont conduit la réflexion sur ce travail, participé à toutes les étapes de la conception et de la réalisation de l'étude et conçu le questionnaire de l'enquête. MarieChristine Leymarie a diffusé l'enquête et réalisé le recueil de données. Christine Morin a effectué l'analyse des données. Les deux auteurs ont contribué à la révision scientifique du contenu et à l'approbation de la version finale.

\section{Remerciements}

Les auteurs remercient le Dr Atf Ghérissi (Tunisie), le Dr Marianne Mead (Royaume Uni), sages-femmes enseignant-chercheurs, Françoise Nguyen (France), présidente de la CNEMa (20052013) pour les conseils qu'elles ont apportés et l'ensemble des sages-femmes enseignantes et directrices qui ont accepté de répondre à l'enquête.

\section{Déclaration d'intérêts}

Les auteurs déclarent ne pas avoir de conflits d'intérêt en lien avec le contenu de cet article. 


\section{Approbation éthique}

En accord avec la législation française au moment de la mise en œuvre de l'enquête, aucune démarche d'approbation éthique n'a été nécessaire puisque l'enquête s'adressait à des professionnels. Cependant, les membres des équipes pédagogiques ont été informés des procédures mises en œuvre pour assurer la protection et l'utilisation des données ainsi que de la possibilité de refuser de participer à l'enquête. Une anonymisation des écoles et des noms a été effectuée afin de garantir la confidentialité des informations.

\section{Références}

1. Sleep J, Grant A. West Berkshire perineal management trial: three year follow up. Br Med J (Clin Res Ed) 1987;295(6601):749-51.

2. Stamp GE. Care of the perineum in the second stage of labour: A study of views and practices of Australian midwives. Midwifery 1997;13:100-4.

3. Gross MM, Hecker H, Matterne A, Guenter HH, Keirse MJNC. Does the way that women experience the onset of labour influence the duration of labour? BJOG 2006;113:289-94.

4. Sandall J, Soltani H, Gates S, Shennan A, Devane D. Midwife-led continuity models versus other models of care for childbearing women. Cochrane Database Syst Rev 2013;8:CD004667.

5. Ten Hoope-Bender P, and coll. Improvement of maternal and newborn health through midwifery. The Lancet 2014;384:1226-35.

6. European perinatal health report. Health and care of pregnant women and babies in Europe in 2010. 2010 [On-line] Disponible sur: http://www.europeristat.com

7. Thompson JE. Competencies for midwifery teachers. Midwifery 2002;18:25-9.

8. Renfrew MJ, McFadden A, Bastos MH, Campbell J, Channon AA, Cheung NF, et al. Midwifery and quality care: findings from a new evidence-informed framework for maternal and newborn care. The Lancet 2014;384:1129-45.
9. Homer CSE, Friberg IK, Dias MAB, ten Hoope-Bender P, Sandall J, Speciale AM, et al. The projected effect of scaling up midwifery. The Lancet 2014;384:1146-57.

10. Knibiehler Y. Accoucher. Femmes, sages-femmes et médecins depuis le milieu du XXe siècle. Rennes : Presses de l'EHESP; 2 ème Edition, 2016

11. Morin C. Histoire de l'amélioration de la mortalité maternelle et du rôle des sages-femmes françaises. In: Les sages-femmes dans la francophonie Témoins silencieuses de la vie. et de la mort. Edition du CHU Sainte-Justine, 2013:259-64.

12. Schweyer F-X. La profession de sage-femme : autonomie au travail et corporatisme protectionniste. Sciences sociales et santé 1996;14:67-102.

13. Journal officiel de la République française. Loi $n^{\circ}$ 2009-879 du 21 juillet 2009 portant réforme de l'hôpital et relative aux patients, à la santé et aux territoires.

14. International Confederation of Midwives (ICM). Définition Internationale de la Sage-femme. 2011 [Online] Disponible sur: http://www.internationalmidwives.org/who-we-are/policy-and-practice/icminternational-definition-of-the-midwife/international-definition-of-a-midwife-french.html

15. International Confederation of Midwives (ICM). Compétences essentielles pour la pratique de base du métier de sage-femme, 2013.

16. Thompson JB, Fullerton JT, Sawyer AJ. The International Confederation of Midwives: Global Standards for Midwifery Education (2010) with Companion Guidelines. Midwifery 2011;27(4):409-16.

17. Le Parlement européen et le Conseil. Directive 2005/ 36/CE du Parlement européen et du Conseil du 7 septembre 2005 relative à la reconnaissance des qualifications professionnelles.

18. Le Parlement et le Conseil de l'Union Européen. Directive 2013/55/UE du Parlement européen et du Conseil du 20 novembre 2013 modifiant la directive 2005/36/CE relative à la reconnaissance des qualifications professionnelles.

19. Farley C, Carr KC. New Directions in Midwifery Education: The Master's of Science in Midwifery Degree. The Journal of Midwifery \& Women's Health 2003;48(2):133-7. 
20. Sinclair M. Editorial - Doctoral midwifery: an investment for the profession. Evidence Based Midwifery 2006;4(1):3.

21. Sinclair M. Editorial - The PhD viva: a necessary rite of passage. Evidence Based Midwifery 2007;5(4):111.

22. International Confederation of Midwives (ICM). Sage-femme?: une profession autonome. 2011.

23. Cour des comptes. Rapport de la sécurité sociale 2011?: Le rôle des sages-femmes dans le système de soins. 2011 p. Pages 173-198. Disponible sur: http:/ /www.ccomptes.fr/Publications/Publications/Securite-sociale-2011

24. Nguyen F. Formation initiale sage-femme : enjeux et perspectives. Création d'une filière sage-femme à l'université. Projet d'application de la réforme LMD au cursus sage-femme. La Revue Sage-Femme 2008;7(1):38-41.

25. République française. Arrêté du 19 juillet 2011 relatif au régime des études en vue du diplôme de formation générale en sciences maïeutiques.

26. République Française. Arrêté du 11 mars 2013 relatif au régime des études en vue du diplôme d'Etat de sage-femme.

27. République française. Arrêté du 28 octobre 2009 relatif à la première année commune aux études de santé.

28. Leymarie M-C, Demeester A. Un cursus universitaire pour la formation des sages-femmes: Discussion et propositions. La Revue Sage-Femme 2006;5(5): 266-73.

29. Henart L, Berland Y, Cadet D. Rapport relatif aux métiers en santé de niveau intermédiaire, 2011.

30. République Française. Code de la santé publique Livre Ier?: Professions médicales. Article L4111-1 à L4127-1.

31. Leymarie M-C. Intégration universitaire des écoles de sages-femmes. La Revue Sage-Femme 2010;9(4): 211-8.

32. République française. Arrêté du 11 septembre 2009 relatif aux modalités d'agrément des écoles de sagesfemmes et de cadres sages-femmes.

33. Demeester A. Formation initiale sage-femme : l'université est une chance. Saisissons-la ! La Revue Sage-Femme 2011;10(3):101-3.

34. République Française. Code de l'éducation - Dispositions propres aux unités de formation et de recherche de médecine, pharmacie, odontologie et maïeutique. Article L713-4 À Article L713-8.

35. Inspection Générale des Affaires Sociales (IGAS), Inspection générale de, l'administration de l'éducation, nationale et de la recherche. Evaluation de l'impact du dispositif sur les formations et le statut des professions paramédicales. 2008.

36. République française. Décret $n^{\circ} 90-949$ du 26 octobre 1990 portant statut particulier des directeurs d'école de sages-femmes de la fonction publique hospitalière.

37. Schytt E, Waldenström U. How well does midwifery education prepare for clinical practice? Exploring the views of Swedish students, midwives and obstetricians. Midwifery 2013;29(2):102-9.

38. The nursing and midwifery professoriate in the UK [Internet]. The Royal College of Nursing. Disponible sur: /professional-development/research-and-innovation/research-training-and-careers/the-nursingprofessoriate-in-the-uk

39. Skirton H, Stephen N, Doris F, Cooper M, Avis M, Fraser DM. Preparedness of newly qualified midwives to deliver clinical care: an evaluation of preregistration midwifery education through an analysis of key events. Midwifery 2012;28(5):e660-666.

40. République Française. Décret $\mathrm{n}^{\circ}$ 2010-804 du 13 juillet 2010 relatif aux missions de l'Observatoire national de la démographie des professions de santé.

41. République Française. Décret n ${ }^{\circ} 2015-813$ du 3 juillet 2015 relatif à la Commission nationale des études de maïeutique, médecine, odontologie et pharmacie.

42. République française. Décret $n^{\circ} 2010-762$ du 7 juillet 2010 relatif à la commission pédagogique nationale des études de santé.

43. Naiditch M. La sage-femme face aux pouvoirs?: une analyse politique de la grève des SF comme outil de combat pour une pleine reconnaissance de leur profession. 2014

Disponible sur: http://www.jesuislasagefemme.com/ jesuislasagefemme/les_interventions_2014_files/ Michel\%20Naiditch.pdf

44. Gaillard R. Rapport Mission sur l'évolution du statut hospitalo-universitaire. 2011.

45. International Confederation of Midwives (ICM). Normes globales de formation des sages-femmes. [Internet]. 2013 [cité 13 déc 2014]. Disponible 
sur: http://internationalmidwives.org/global/fran-

cais/formation-les-normes.html

46. Metté C, Leroux C, Gagnayre R. Détermination de critères/indicateurs d'évaluation de la compétence pédagogique par une enquête d'opinion auprès d'enseignants chercheurs de l'UFR de médecine de Bobigny (Université Paris 13). Pédagogie médicale 2005;6(1):32-41.

47. Morin C. Développer la recherche dans la formation initiale des sages-femmes. La Revue Sage-Femme 2010;9(5):253-9.

48. Fleming VEM, Hagen S, Niven C. Does perineal suturing make a difference? The SUNS trial. BJOG 2003;110(7):684-9.

49. Fraser DM, Avis M, Mallik M. The MINT project-An evaluation of the impact of midwife teachers on the outcomes of pre-registration midwifery education in the UK. Midwifery 2013;29(1):86-94.

50. Conseil National de l'Ordre des sages-femmes. Des docteurs chez les sages-femmes 2010;(23):8.

51. Mead M. Editorial -Similarités et différences politiques et socio-culturelles entre la France, la Suède et le Royaume Uni. Les Dossiers de la Maïeutique 2014;1(2):43-9.

52. Royal College of Nursing. Trends in the UK nursing and midwifery professions' professoriate over the last ten years Disponible sur:

http://www2.rcn.org.uk/development/

research_and_innovation/career/

nursing_professoriate/professoriate_survey_2013

53. Gagnon R. Midwifery in a new context: Expanding our reference points and embracing new representations of pregnancy and birth. Midwifery 2011;27(3): 360-7.

54. Maillefer F, de Labrusse C, Cardia-Vonèche L, Hohlfeld P, Stoll B. Women and healthcare providers' perceptions of a midwife-led unit in a Swiss university hospital: a qualitative study. BMC Pregnancy Childbirth 2015;15:56.

55. de Labrusse C, Ramelet A-S, Humphrey T, Maclennan SJ. Patient-centered Care in Maternity Services: A Critical Appraisal and Synthesis of the Literature. Women's Health Issues 2016;26(1):100-9.

56. Gourounti K, Sandall J. Do pregnant women in Greece make informed choices about antenatal screening for
Down's syndrome? A questionnaire survey. Midwifery 2008;24(2):153-62.

57. School of Community Health and Midwifery [Internet]. UCLan - University of Central Lancashire. Disponible sur: http://www.uclan.ac.uk/schools/ community-health-midwifery/index.php

58. King's College London - Florence Nightingale Faculty of Nursing \& Midwifery Disponible sur: http://www.kcl.ac.uk/nursing/Index.aspx

59. République Française. Code de la santé publique Article D4151-10.

60. Perrier I, Vendittelli F. Devenir et impact sur les pratiques professionnelles des mémoires des étudiants sages-femmes. La Revue Sage-Femme 2010;9(4): 165-72.

61. Ministère de l'enseignement supérieur et de la Recherche. Diplôme d'État de sage-femme. Bulletin officiel $\mathrm{n}^{\circ} 15$ avr 11, 2013.

62. ICM. Compétences Essentielles pour la Pratique de Base du Métier de Sage-femme (Révisé 2013) Disponible sur: http://www.internationalmidwives.org/ global/francais/

63. République française. Code de déontologie des sagesfemmes. Articles R4127-301 à 367.

64. Shaw D, Guise J-M, Shah N, Gemzell-Danielsson K, Joseph K, Levy B, et al. Drivers of maternity care in high-income countries: can health systems support woman-centred care? The Lancet 2016;388(10057): 2282-95.

65. Chamberland M, Hivon R. Les compétences de l'enseignant clinicien et le modèle de rôle en formation clinique. Pédagogie médicale 2005;6(2):98-111.

66. Laflamme S. Un service d'aide aux étudiants offert par des enseignants experts?: la réussite de l'étudiant au cœur des préoccupations. Pédagogie Médicale 2014;15(1):43-52.

67. République Française. Code de l'éducation - Les formations de santé - Article L631-1 à L636-1.

Correspondance et offprints : Christine MORIN : Ecole de sagesfemmes de Bordeaux, Centre hospitalier Pellegrin Place Amélie Raba-Léon, 33076 Bordeaux Cedex, France.

Mailto : christine.morin@chu-bordeaux.fr 